\title{
The Sun as the Source of Nonlinear MHD Perturbations of the Solar Corona and the Heliosphere
}

\author{
S.A. Grib \\ Academy of Science, Central (Pulkovo) Astronomical Observatory, \\ 196140, 61/1, Pulkovskoe Shosse, Saint-Petersburg, Russia
}

\begin{abstract}
The extended discussion of the Nonlinear MHD Perturbations of the Solar Corona and the Heliosphere has been exposed.
\end{abstract}

As it is known nowadays especially from the results of SOLTIP project many nonlinear perturbations appearing inside the solar corona and the solar wind are interconnected with different magnetohydrodynamic (MHD) waves and discontinuities. Obviously the MHD approach to the study of the solar corona and the heliosphere is limited by the continuum approximation but it gives often an opportunity to learn the values of many macroscopic parameters, which describe the dynamics of the solar corona and the solar wind with their stationary and nonstationary phenomena. It's possible in the frame of MHD to describe an oblique collision between different MHD discontinuities as the splitting of an arbitrary discontinuity and to change the problem to a stationary one using afterwards the generalized polars (Barmin A.A. and Pushkar E.A. 1990). It is reasonable to take the specific model of the MHD contact discontinuity $\mathrm{C}$ connected with the transition region going from the solar chromosphere to the corona across which we have an abrupt increase of the plasma temperature and a decrease of its density (Hansteen V.H. 2001). So we may have A C interaction for the solar rotational or Alfven discontinuity A. Using a definite angle between the magnetic field and the contact discontinuity as 60 degrees with a change of an angle from 165 to 175 degrees for A colliding with $\mathrm{C}$ we have the so called catastrophic reconstruction:

$$
A C \rightarrow R_{+} S_{-} C S_{-} A R_{+} \rightarrow R_{+} A S_{-} C S_{-} A R_{+} \rightarrow R_{+} A R_{-} C S_{-} A R_{+} \rightarrow S_{+} R_{-} C R_{-} A S_{+}
$$

where $R_{+}, R_{-}, S_{+}, S_{-}$are fast and slow rarefaction and shock waves. Thus the solar rotational discontinuities linked with Alfven waves may refract as a dissipative shock waves and they will heat the coronal plasma. This point was at first declared by Grib and Koutchmy (2001). The discontinious structure is important for many pressure balanced structures (Burlaga L.F. 1995) and sometimes they have an anisotropy of pressure (Vasquez A.M., van Ballegooijen A.A., and Raymond J.C. 2003). It is important to indicate that the thermal anisotropy may be increased in the vicinity of the fast plasma flows in the solar corona across the solar shock wave fronts (Grib S.A. and Hrapov B.A. 1989). This work was done in the frame of the OFN RAN program 18.

\section{Summary}

The rotational solar discontinuity may create the dissipative coronal shock wave heating the coronal plasma. The quasi MHD solar shock waves may effect the proton thermal anisotropy observed recently in the coronal holes plasma. 


\section{References}

Barmin A.A., Pushkar E.A. 1990, The oblique interaction of Alfven and contact discontinuities in MHD. Mec.of Fluids and Gas, 1, 131-142

Hansteen V.H. 2001, Key issues of the solar transition region to be addressed by the Solar Orbiter. Proc. of Solar Encounter. The First Solar Orbiter Workshop. Puetro de la Cruz. Tenerife, Spain, 14-18 May 2001. CESA SP-494, Sept. 2001, 51-58

Grib S.A., Koutchmy S. 2001, The dissipation of a magnetic field energy in the coronal MHD discontinuities and in the solar wind strong discontinuities. The Sun during the epoch of the change of magnetic field sign. Proc.of the Int.Conf. GAO RAN. Saint-Petersburg. 2001 $149-154$

Burlaga L.F. 1995, Interplanetary Magnetohydrodynamics (NY Oxford Univ. Press)

Vasquez A.M., van Ballegooijen A.A., Raymond J.C. 2003, The effect of proton temperature anisotropy in the solar minimum corona and wind. Astroph. J., 598, 2, p.1, 1361-1374

Grib S.A., Hrapov B.A. 1989, The change of anisotropic space plasma parameters across the front of the MHD shock wave. Space Res., 27, 2, 258-266 